\title{
2013 Reviewers
}

\begin{tabular}{|c|c|c|}
\hline Shannon Zenk & Danielle C. Ompad & Sonia Jain \\
\hline George Woody & Carolyn Olson & Richard J. Jackson \\
\hline Amy Funk Wolkin & Allison J. Ober & Mark Huntington \\
\hline Kathleen Wirth & LaRon E. Nelson & Lisa Henriksen \\
\hline Garen Wintemute & Kenrad Nelson & Trudy Harpham \\
\hline Dan Werb & Neo Morojele & Cheryl Hanna \\
\hline Cyprian Wejnert & Mark Randolph & Ellie Grossman \\
\hline Linda Weiss & Montgomery & Ruth Greenblatt \\
\hline Billie Weiss & Geoffrey Monaghan & Emily Goldmann \\
\hline Daniel Webster & Rachel Miller & Robyn R. M. Gershon \\
\hline Eve Waltermaurer & Elizabeth Miller & Kirti Gaur \\
\hline Kate Walsh & Lynne Messer & Andrea Gartner \\
\hline Arpana Verma & Jacqueline Merrill & Robert Fullilove \\
\hline Tom Valente & Judith McFarlane & Crystal Fuller \\
\hline Marcelo Luis Urquia & Donna H. McCree & Elena Fuentes-Afflick \\
\hline Benita Tsao & Susan Mason & Victoria Frye \\
\hline Emmanuel Thomas & Alex F. Manini & Nicholas Freudenberg \\
\hline Richard Tewksbury & Mary Mackesy-Amiti & Ruth Finkelstein \\
\hline John Song & Gina Schellenbaum & Don Des Jarlais \\
\hline Dawn K. Smith & Lovasi & Carol Dawson-Rose \\
\hline Susan Sherman & Brett R. Loomis & Peter Davidson \\
\hline Holly Shakya & TK Logan & Shane Darke \\
\hline Maria Paula Maia & Weiwei Liu & Nihaya Daoud \\
\hline Santos & Yeonjung Lee & Jason Corburn \\
\hline Glenn-Milo Santos & Joshua Lee & Hannah Cooper \\
\hline Mojgan Sami & Carl Latkin & Phillip O Coffin \\
\hline Abby E. Rudolph & Alex Kral & Adam Carrico \\
\hline Elise D. Riley & James Kirby & Waleska Caiaffa \\
\hline Marilyn Rice & Kunal Keshri & Jessica Burke \\
\hline Kurt Ribisl & Deanna Kerrigan & Barbara Jean Burgel \\
\hline Elizabeth Reed & Jacqueline Kerr & Judith S. Brook \\
\hline Naomi Priest & Adam Karpati & Arline Bronzaft \\
\hline Carmen Portillo & Andrew Kanter & Amy Bohnert \\
\hline Roberto Pereira & Michael Johns & Deborah Azrael \\
\hline Joseph Palamar & Jacky M. Jennings & Michael Patrick Arnold \\
\hline David Ostrow & Evelyn Angel James & Mieke Aarts \\
\hline
\end{tabular}

